\title{
Missile-Borne SAR Raw Signal Simulation for Maneuvering Target
}

\author{
Weijie Xia, Yuanyuan Qi, Linlin Huang, and Xue Jin \\ College of Electronic and Information Engineering, Nanjing University of Aeronautics and Astronautics, Nanjing 211100, China \\ Correspondence should be addressed to Weijie Xia; nuaaxwj@nuaa.edu.cn
}

Received 25 February 2016; Revised 28 April 2016; Accepted 10 May 2016

Academic Editor: Atsushi Mase

Copyright (C) 2016 Weijie Xia et al. This is an open access article distributed under the Creative Commons Attribution License, which permits unrestricted use, distribution, and reproduction in any medium, provided the original work is properly cited.

\begin{abstract}
SAR raw signal simulation under the case of maneuver and high-speed has been a challenging and urgent work recently. In this paper, a new method based on one-dimensional fast Fourier transform (1DFFT) algorithm is presented for raw signal simulation of maneuvering target for missile-borne SAR. Firstly, SAR time-domain raw signal model is given and an effective Range Frequency Azimuth Time (RFAT) algorithm based on 1DFFT is derived. In this algorithm, the "Stop and Go" (SaG) model is adopted and the wide radar scattering characteristic of target is taken into account. Furthermore, the "Inner Pulse Motion" (IPM) model is employed to deal with high-speed case. This new RFAT method can handle the maneuvering cases, high-speed cases, and bistatic radar cases, which are all possible in the missile-borne SAR. Besides, this raw signal simulation adopts the electromagnetic scattering calculation so that we do not need a scattering rate distribution map as the simulation input. Thus, the multiple electromagnetic reflections can be considered. Simulation examples prove the effectiveness of our method.
\end{abstract}

\section{Introduction}

Synthetic aperture radar (SAR) raw signal generation and image simulation $[1,2]$ play a significant role in the development of SAR system. On the one hand, SAR raw signal generation is an effective and financial tool that enables us to obtain the echo data needed for the validation of the radar imaging algorithms; on the other hand, SAR image simulation provides a feasible way to establish a target feature database, which is the foundation of the target automatic recognition.

Because of the importance of SAR echo and image simulation, a lot of researches focused on the field have been carried out. In the published literature, approaches to simulating SAR echo signal can be divided into three categories: timedomain (TD) methods, two-dimensional (2D) frequency domain methods, and hybrid time/frequency (TF) domain methods [3]. Classical TD method was once employed by Mori to present an interferometric SAR simulator [4]. This algorithm has a high precision but is time consuming, and the scattering characteristic of the whole target is difficult to take into account. To improve the processing efficiency, the $2 \mathrm{D}$ method, taking advantage of the efficiency of fast Fourier transform algorithm, has been studied in many papers [58]. Unfortunately, this method cannot be directly employed under the situation of nonideal trajectory. As a tradeoff of the aforementioned two methods, hybrid TF simulation, with the ability to consider both the nonideal trajectory and the moderate computational efficiency, has also been an effective way to operate SAR simulation $[9,10]$.

So far, quite a lot of studies have been addressed to simulate SAR raw data [11-16]. However, unlike the traditional SAR configuration in which the platform travels an ideal trajectory, for realistic missile-borne SAR system, both the platform and the target move along curvilinear trajectories with acceleration and even jerk, which may result in twoorder even high-order terms in the range history. With this special range history, the traditional approaches cannot be directly adopted to obtain its $2 \mathrm{D}$ frequency spectrum for the overall SAR system transfer function which depends on the azimuth and range coordinates of the target. Consequently, the improved SAR raw signal simulator with the ability to consider the curvilinear trajectory is strongly required. With respect to this, Franceschetti et al. [11] proposed an efficient 
SAR raw signal simulator based on full 2D Fourier transform, but this simulator is only effective for cases of narrow beam and slow trajectory deviation. Meng et al. developed a fast raw data simulator based on the effectual access of 2DFS [17], but there was still phase space variance which could lead to the inaccurateness of the raw signal. Besides, to our knowledge, the most important feature of the complex target, namely, the dynamic Radar Cross Section (RCS), has not been introduced to the echo generation so far in the literature, and available methods often need to know discrete reflectivity distribution map or digital elevation model (DEM) of the imaged scene as an input. Furthermore, there has not been an integrated method for dynamic target echo simulation which can deal with both monostatic SAR and bistatic SAR in the published literatures.

Aiming at the above problems, in this paper, we will fully take the target RCS feature into account to propose a novel RFAT echo generation method for missile-borne SAR. In this method, the high frequency electromagnetic scattering calculation method based on physical optics (PO), geometrical optics (GO), and Incremental Length Diffraction Coefficients (ILDC) is adopted to calculate the scattering characteristic of target; the SaG model and the IPM model are conducted into the real-time motion simulation; the 1DFFT based echo simulation method is used to generate SAR raw data; finally, the RDA (range-Doppler algorithm) is adopted to obtain the simulated SAR image; simulation results show that the method is effective and suitable for different kinds of SAR (monostatic and bistatic) and motion situations.

\section{Analysis of the Proposed Simulation Algorithm}

2.1. Signal Model. In this section, the main employed symbols (nomenclature) are defined as follows.

$f_{0}$ : carrier frequency.

c: light speed.

$\lambda$ : carrier wavelength.

$T_{r}$ : chirp duration.

$R$ : target-to-antenna distance.

$x_{n}$ : sensor position azimuth coordinate at $t_{n}$.

$k$ : chirp rate.

$t_{n}$ : slow time.

$t^{\prime}$ : fast time.

$N_{a}$ : number of slow time samples.

$N_{r}$ : number of fast time samples.

$x^{\prime}$ : continuous form of $x_{n}$.
Supposing that the radar is illuminating a point scatterer $P$ with a series of chirp signals, the received raw data for $P$ can be described by the following expression:

$$
\begin{aligned}
& s\left(t, t_{n}, R\right)=\gamma \cdot \operatorname{rect}\left[\frac{t-t_{n}-2 R / c}{T_{r}}\right] \\
& \cdot \exp \left[-j 2 \pi f_{0} \frac{2 R}{c}+j \pi k\left(t-t_{n}-\frac{2 R}{c}\right)^{2}\right],
\end{aligned}
$$

where $\gamma$ is the reflectivity function. For simplicity, replacing $t-t_{n}$ in (1) by $t^{\prime}$, we obtain

$$
\begin{aligned}
& s\left(t^{\prime}, x_{n}, R\right)=\gamma \cdot \operatorname{rect}\left[\frac{c t^{\prime} / 2-R}{c T_{r} / 2}\right] \\
& \cdot \exp \left[-\frac{j 4 \pi R}{\lambda}+\frac{j 4 \pi k}{c^{2}}\left(\frac{c t^{\prime}}{2}-R\right)^{2}\right],
\end{aligned}
$$

where $c t^{\prime} / 2$ is the spatial sampling interval in the range direction; let $r^{\prime}=c t^{\prime} / 2$; the echo equation of the point scatterer in the range-time domain can be written as follows:

$$
\begin{aligned}
& s\left(x_{n}, r^{\prime}, R\right) \\
& =\gamma \\
& \quad \cdot \operatorname{rect}\left[\frac{r^{\prime}-R}{c T_{r} / 2}\right] \exp \left[-j \frac{4 \pi R}{\lambda}+j \pi k \frac{4}{c^{2}}\left(r^{\prime}-R\right)^{2}\right] .
\end{aligned}
$$

Actually, the complex target consists of many point scatterers; the echo signal of the target can be calculated by summing up each point scatterer echo signal as follows:

$$
\begin{aligned}
& s\left(x^{\prime}, r^{\prime}\right)=\int \gamma\left(x^{\prime}, R\right) \text { rect }\left[\frac{r^{\prime}-R}{c T_{r} / 2}\right] \exp \left(-j \frac{4 \pi R}{\lambda}\right) \\
& \cdot \exp \left[j \pi k \frac{4}{c^{2}}\left(r^{\prime}-R\right)^{2}\right] d R,
\end{aligned}
$$

where $\gamma\left(x^{\prime}, R\right)$ is the overall reflectivity of all points at distance $R$ from the sensor. Through (4), we can simulate the SAR raw data in the time domain directly, but it suffers from the disadvantages of high computation complexity. Furthermore, it is hard to obtain the scattering center points of a complex target. To solve this problem, a Fourier transform is implemented in the range direction to (4):

$$
\begin{aligned}
& S\left(x^{\prime}, \eta\right)=\int s_{r}\left(x^{\prime}, r^{\prime}\right) \exp \left(-j \eta r^{\prime}\right) d r^{\prime}=\iint \gamma\left(x^{\prime}, R\right) \\
& \cdot \exp \left(-j \frac{4 \pi R}{\lambda}\right) \exp \left[j \pi k \frac{4}{c^{2}}\left(r^{\prime}-R\right)^{2}\right] \\
& \cdot \operatorname{rect}\left[\frac{r^{\prime}-R}{c T_{r} / 2}\right] \exp \left(-j \eta r^{\prime}\right) d R d r^{\prime}
\end{aligned}
$$




$$
\begin{aligned}
& =\int \gamma\left(x^{\prime}, R\right) \exp \left(-j \frac{4 \pi R}{\lambda}\right) \\
& \cdot \int \exp \left[j \pi k \frac{4}{c^{2}}\left(r^{\prime}-R\right)^{2}\right] \operatorname{rect}\left[\frac{r^{\prime}-R}{c T_{r} / 2}\right] \\
& \cdot \exp \left(-j \eta r^{\prime}\right) d r^{\prime} d R=\int \gamma\left(x^{\prime}, R\right) \\
& \cdot \exp \left(-j \frac{4 \pi R}{\lambda}\right) \exp (-j \eta R) \operatorname{rect}\left[\frac{c \eta}{4 k \pi T_{r}}\right] \\
& \cdot \exp \left(-j \frac{\eta^{2} c^{2}}{16 k \pi}\right) d R=\operatorname{rect}\left[\frac{c \eta}{4 k \pi T_{r}}\right] \exp (-j \\
& \left.\cdot \frac{\eta^{2} c^{2}}{16 k \pi}\right) \int \gamma\left(x^{\prime}, R\right) \exp \left[-j\left(\frac{4 \pi}{\lambda}+\eta\right) R\right] d R \\
& =\operatorname{rect}\left[\frac{c \eta}{4 k \pi T_{r}}\right] \exp \left(-j \frac{\eta^{2} c^{2}}{16 k \pi}\right) \Gamma\left[x^{\prime},\left(\frac{4 \pi}{\lambda}+\eta\right)\right],
\end{aligned}
$$

where $\Gamma\left[x^{\prime},(4 \pi / \lambda+\eta)\right]$ represents the Fourier transformation of the scattering coefficient of the target which will be explained specifically in the next section and $\eta$ refers to the corresponding spatial frequency of the range coordinate variable $r^{\prime}$ with the unit of $\mathrm{rad} / \mathrm{m}$. According to the stretchable nature of the Fourier transform, the relationship between range frequency and spatial frequency can be described as

$$
\begin{gathered}
f_{r}=\frac{c}{4 \pi} \eta, \\
\text { or } \eta=2 \pi \frac{2 f_{r}}{c} .
\end{gathered}
$$

Substituting (6) to (5), we obtain

$$
\begin{aligned}
S\left(x^{\prime}, f_{r}\right)= & \operatorname{rect}\left[\frac{f_{r}}{k T_{r}}\right] \exp \left[-j \frac{\pi f_{r}^{2}}{k}\right] \\
& \cdot \Gamma\left\{x^{\prime},\left[\frac{4 \pi\left(f_{0}+f_{r}\right)}{c}\right]\right\},
\end{aligned}
$$

which is the echo equation in the range frequency domain. Then, the original time-domain echo signal can be obtained after a $1 D$ inverse Fourier transform.

2.2. Dynamic Modeling for Electromagnetic Scattering. From the final expression described in (7), we can find that the key procedure of generating the echo data is the computation of $\Gamma\left\{x^{\prime}, f_{0}+f_{r}\right\}$. As mentioned in the previous section, $\Gamma\left\{x^{\prime}, f_{0}+\right.$ $\left.f_{r}\right\}$ is the Fourier transformation of the scattering coefficient, namely, the Radar Cross Section (RCS) of the target. Thus, in this section, the PO, GO, and ILDC based RCS calculation method is first introduced and then the Doppler correction method for dynamic target is given.

2.2.1. The $P O / G O$ and ILDC Method. The GO is a ray-based method intended for the consideration of electrically large structures, which employs ray-launching and transmission, reflection, and refraction theory to model the interaction between the dielectric regions [18]. The PO utilizes ray optics to estimate the field on a surface and then calculate the transmitted or scattered field through Stratton-Chu formulation [19]. The backscatter contributions are evaluated by both GO and $\mathrm{PO}$, which can be used to compute multiple reflections effectively. Furthermore, the edge diffraction effect of electrically large scaled complex objects is also considered and calculated with the theory of Incremental Length Diffraction Coefficients (ILDC) [20].

2.2.2. Doppler Correction Method. For dynamic missile target, the distance change between target and radar over time could lead to the Doppler effects in the echo [21]. Thus, it is needed for modified results of RCS calculation to take Doppler effects into account.

The modified RCS can be expressed as

$$
\Gamma_{1}(f, t)=\exp \left[\frac{-j 4 \pi R^{c}(t) f}{c}\right] \Gamma_{0}(f, t) .
$$

In this formula, $R^{c}(t)$ represents the distance from the geometric center of the complex target to the sensor, $\exp \left(-j 4 \pi R^{c}(t) f / c\right)$ is the Doppler phrase term, $f$ denotes the electromagnetic wave frequency, and $\Gamma_{0}(f, t)$ and $\Gamma_{1}(f, t)$ are the RCS before phrase correction and the modified RCS, respectively.

2.3. Dynamic Target Range History Analysis. In order to realize the phase correction described in (8), dynamic target range history analysis is presented in this section.

2.3.1. Monostatic Radar. Consider the missile-borne SAR illuminating an aerial maneuvering target as shown in Figure 1. The missile-borne SAR platform denoted by $\vec{Q}_{0}$ moves along the nonideal trajectory $\vec{Q}(t)$ which obeys a high-order polynomial-type representation as a function of the continuous time:

$$
\vec{Q}(t)=\vec{Q}_{0}+\vec{v}_{q} t+\vec{a}_{q} t^{2}+\vec{g}_{q} t^{3}+\cdots,
$$

where $\vec{v}_{q}$ is the initial velocity vector, $\vec{a}_{q}$ is the initial acceleration vector, and $\vec{g}_{q}$ is the jerk vector. Similarly, the target denoted by $\vec{P}_{0}$ moves along the nonideal trajectory $\vec{P}(t)$ which has the following expression:

$$
\vec{P}(t)=\vec{P}_{0}+\vec{v}_{p} t+\vec{a}_{p} t^{2}+\vec{g}_{p} t^{3}+\cdots,
$$

where $\vec{v}_{p}, \vec{a}_{p}$, and $\vec{g}_{p}$ are the initial velocity vector, the acceleration vector, and the jerk vector, respectively.

Then, the instantaneous slant range between target and radar can be expressed as

$$
\begin{aligned}
R^{c}(t) & =|\vec{Q}(t)-\vec{P}(t)| \\
& =\left|\vec{r}_{0}+\vec{v}_{p q} t+\vec{a}_{p q} t^{2}+\vec{g}_{p q} t^{3}+\cdots\right|,
\end{aligned}
$$




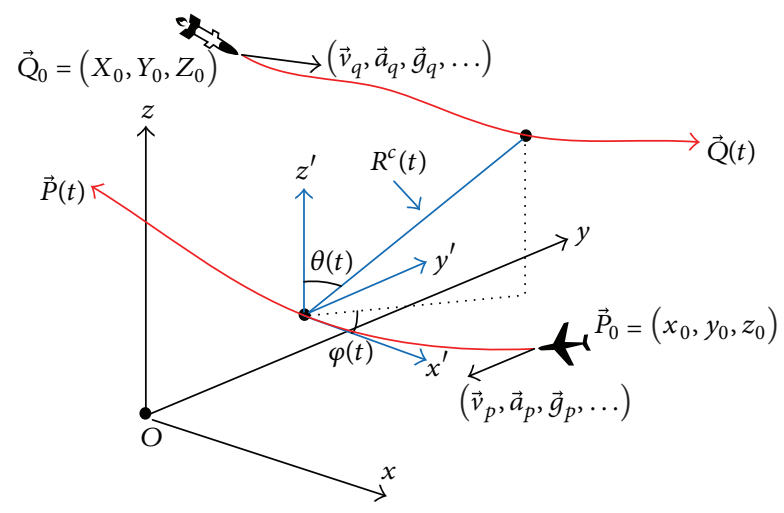

FIgURE 1: Monostatic missile-borne SAR geometry.

where $\vec{r}_{0}=\vec{Q}_{0}-\vec{P}_{0}$ represent the initial vector between radar $\vec{Q}$ and target $\vec{P}$ and $\vec{v}_{p q} \vec{a}_{p q}$, and $\vec{g}_{p q}$ refer to the relative velocity, acceleration, and jerk vector between radar and the target, respectively.

For the situation with a low relative speed, "Stop and Go" (SaG) models are generally employed to describe the motion state. As a quasistatic approximate method, this model supposes that the platform remains stationary between transmitting and receiving pulse. Figure 2 shows the simple graph of the instantaneous distance between radar and target, where the blue line represents the real-time distance and the red line is the $\mathrm{SaG}$ distance which can be written as

$$
\begin{aligned}
R_{\mathrm{sa}}^{c}(t) & =R^{c}\left(t_{n}\right) \\
=\left|\vec{r}_{0}+\vec{v}_{p q} t_{n}+\vec{a}_{p q} t_{n}{ }^{2}+\vec{g}_{p q} t_{n}{ }^{3}+\cdots\right|, & \\
& \quad t \in\left[t_{n}-\frac{T_{r}}{2}, t_{n}+\frac{T_{r}}{2}\right] .
\end{aligned}
$$

Then, the SaG echo signal for a simple point target can be obtained by substituting (12) into (2):

$$
\begin{aligned}
& s_{\mathrm{sa}}\left(t_{n}, R^{c}\right)=\gamma \cdot \operatorname{rect}\left[\frac{t-t_{n}-2 R_{\mathrm{sa}}^{c}(t) / c}{T_{r}}\right] \\
& \cdot \exp \left[-j 2 \pi f_{0} \frac{2 R_{\mathrm{sa}}^{c}(t)}{c}\right. \\
& \left.+j \pi k\left(t-t_{n}-\frac{2 R_{\mathrm{sa}}^{c}(t)}{c}\right)^{2}\right] .
\end{aligned}
$$

It can be seen from the type above that the SaG method has a simple form of echo signal which costs low computational expense. However, this hypothesis ignores the distance error caused by the motion of radar and target within the pulse time. This error can be negligible at the case of low speed but could have a significant impact in the high-speed situation which will be analyzed in the following.

Focusing on the error, we can obviously draw the following conclusions.

(1) The error is minimum (0) when taking the value of $\mathrm{SaG}$ distance as that of the real-time distance in the middle of echo pulse signal, that is, when $t^{\prime}=0$.
(2) The error is the maximum when the sample is located at the endpoints of echo pulse signal; namely, $t^{\prime}= \pm T_{r} / 2$. The maximum range error can be expressed as

$$
\Delta R_{\max }=\left|\frac{\vec{v}_{p q} T_{r}}{2}+\vec{a}_{p q}\left(\frac{T_{r}}{2}\right)^{2}+\vec{g}_{p q}\left(\frac{T_{r}}{2}\right)^{3}+\cdots\right|
$$

When the maximum range error is smaller than a distance resolution cell, the impact it caused can be neglected, namely,

$$
\left|\frac{\vec{v}_{p q} T_{r}}{2}+\vec{a}_{p q}\left(\frac{T_{r}}{2}\right)^{2}+\vec{g}_{p q}\left(\frac{T_{r}}{2}\right)^{3}+\cdots\right|<\frac{2 B}{c},
$$

that is, also the restricted condition of the SaG model; namely, the displacement between the target and the platform in half of the pulse time must be less than one range resolution cell.

2.3.2. High-Speed Cases. As mentioned above, the conventional $\mathrm{SaG}$ model is generally suitable under low speed cases, but there may be extreme cases for which nonnegligible error can be caused by such an approximation, for example, those involving very high relative speed between the platform and the target, or for radars exploiting long time pulse waveforms. Therefore, a new motion model with low complexity and small error should be derived to calculate the range history in extreme cases.

Based on (14), it can be concluded that $\Delta R_{\max }$ is determined by the motion parameters $\left(\vec{v}_{p q}, \vec{a}_{p q}, \vec{g}_{p q}\right)$ and the time parameter $T_{r}$. Since the motion parameters are out of control, the only way to reduce the maximum range error lies in the pulse during time $T_{r}$. According to the fast time sampling, we can divide $T_{r}$ into $N_{r}$ parts as shown in Figure 3.

So that the red points described new range history can be written as

$$
\begin{aligned}
& R_{\mathrm{IPM}}^{c}(t) \\
& =\left|\vec{r}_{0}+\vec{v}_{p q} t\left(t_{n}, m\right)+\vec{a}_{p q} t\left(t_{n}, m\right)^{2}+\vec{g}_{p q} t\left(t_{n}, m\right)^{3}\right|, \\
& \quad t\left(t_{n}, m\right)=t_{n}-\frac{T_{r}}{2}+\frac{m T_{r}}{N_{r}}, m=0,1,2, \ldots, N_{r},
\end{aligned}
$$

where $N_{r}$ is the number of fast time sample points, $m$ is the order number, and $R_{\mathrm{IPM}}^{c}(t)$ is the simulation slant range of the new model. From the expression, it can be found that the new model takes the motion in the duration time into consideration, so it is called the Inner Pulse Motion (IPM) model.

2.3.3. Bistatic Radar. Now let us consider the cases of bistatic missile-borne SAR. The geometric configuration is shown in Figure 4.

In this figure, the transmitter denoted by $\vec{Q}$ moves along the nonideal trajectory $\vec{Q}(t)$, the receiver denoted by $\vec{D}$ moves along the trajectory $\vec{D}(t)$, and the target denoted by $\vec{P}$ moves along the trajectory $\vec{P}(t)$; they are all considered to obey 


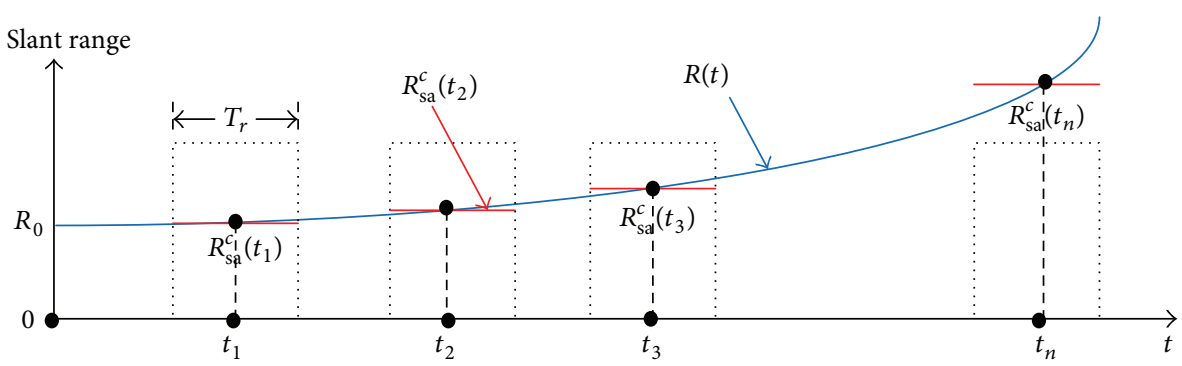

FIgURE 2: Range history of the SaG model.

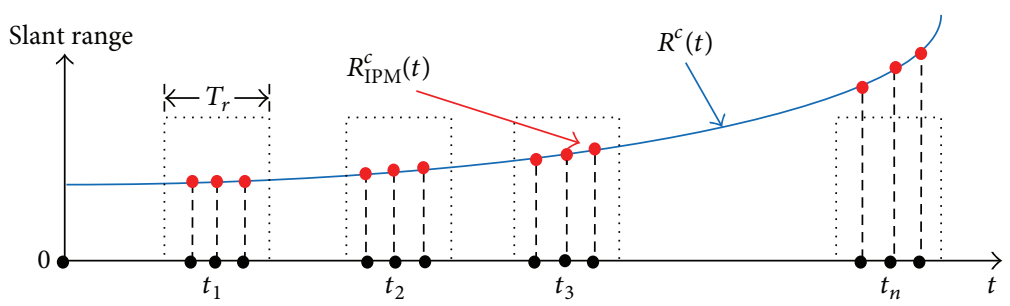

FIgURE 3: Range history of the IPM model.

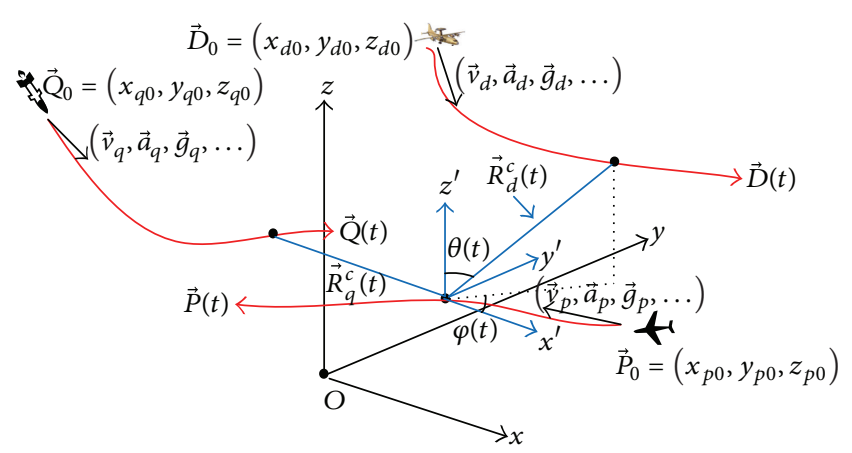

FIGURE 4: Bistatic missile-borne SAR geometry.

a high degree polynomial-type representation as a function of time:

$$
\begin{aligned}
& \vec{Q}(t)=\vec{Q}_{0}+\vec{v}_{q} t+\vec{a}_{q} t^{2}+\vec{g}_{q} t^{3}+\cdots, \\
& \vec{P}(t)=\vec{P}_{0}+\vec{v}_{p} t+\vec{a}_{p} t^{2}+\vec{g}_{p} t^{3}+\cdots, \\
& \vec{D}(t)=\vec{D}_{0}+\vec{v}_{d} t+\vec{a}_{d} t^{2}+\vec{g}_{d} t^{3}+\cdots
\end{aligned}
$$

Then, the equivalent instantaneous slant range can be calculated by summing up the transmitter distance and the receiver distance as follows:

$$
\begin{aligned}
R_{q}^{c}(t) & =|\vec{Q}(t)-\vec{P}(t)| \\
& =\left|\vec{R}_{q 0}^{c}+\vec{v}_{p q} t+\vec{a}_{p q} t^{2}+\vec{g}_{p q} t^{3}+\cdots\right|, \\
R_{d}^{c}(t) & =|\vec{P}(t)-\vec{T}(t)|
\end{aligned}
$$

$$
\begin{aligned}
& =\left|\vec{R}_{d 0}^{c}+\vec{v}_{d p} t+\vec{a}_{d p} t^{2}+\vec{g}_{d p} t^{3}+\cdots\right|, \\
R_{\mathrm{bis}}^{c}(t) & =\frac{\left[R_{d}^{c}(t)+R_{q}^{c}(t)\right]}{2},
\end{aligned}
$$

where $R_{q}^{c}(t)$ is the distance between transmitter and target, $R_{d}^{c}(t)$ is the distance between receiver and target, and $\vec{v}_{p q}$, $\vec{a}_{p q}$, and $\vec{g}_{p q}$ are the relative velocity vector, acceleration vector, and jerk vector between the transmitter and the target, respectively, while $\vec{v}_{d p}, \vec{a}_{d p}$, and $\vec{g}_{d p}$ are those between the target and the receiver.

2.4. Range-Doppler Algorithm. So far, we have completed the echo generation for complicated moving target; next, the typical RDA (range-Doppler algorithm) is applied in this section for data processing. The specific flow diagram is shown as seen in Figure 5.

The SAR image of one point target is simulated to verify the algorithm efficiency. Then, we get Figure 6(a) showing real part of the echo signal; Figure 6(b) shows the range compression results of echo signal; there is one object simulated on the scene, and the range mitigation can be seen clearly. Figure 6(c) shows the time-domain signal after range migration correction, and Figure 6(d) shows the final SAR image after range migration correction and azimuth compression in which the point target can be clearly distinguished.

It must be noted that in this paper we focus our research on the SAR echo and image simulation, not with the SAR imaging algorithm. The RDA is employed here to further verify the results of echo simulation with given parameters of the simulation, while the Doppler parameters estimation and the range cell migration correction (RCMC) it includes are beyond the scope of our research. 


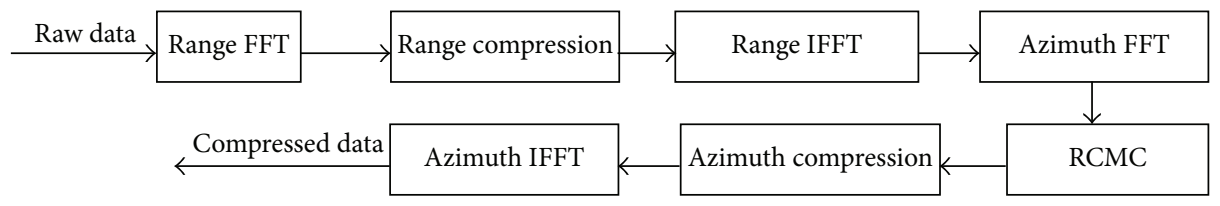

FIgURE 5: Flowchart of RDA.

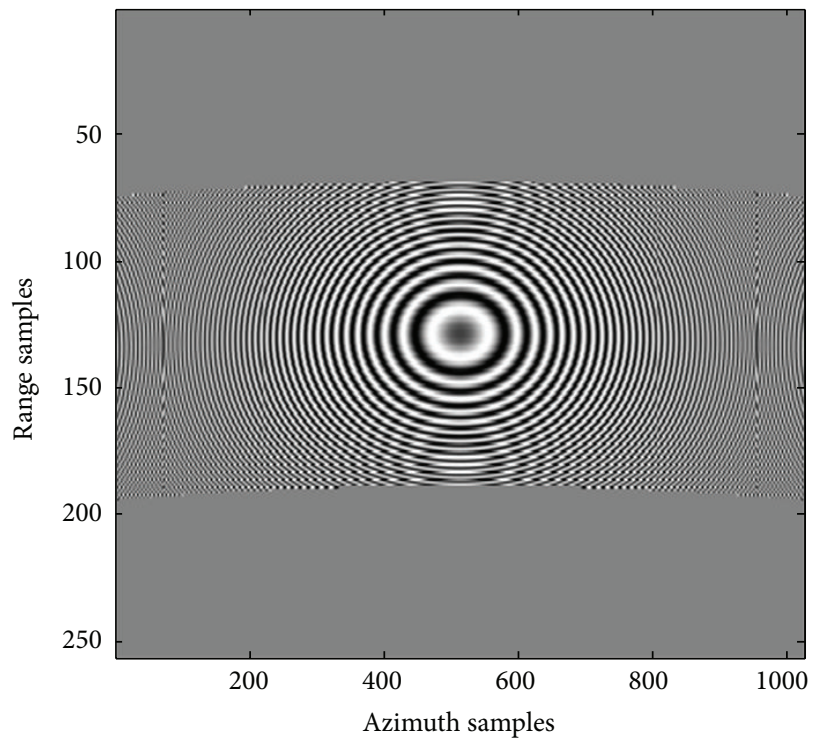

(a)

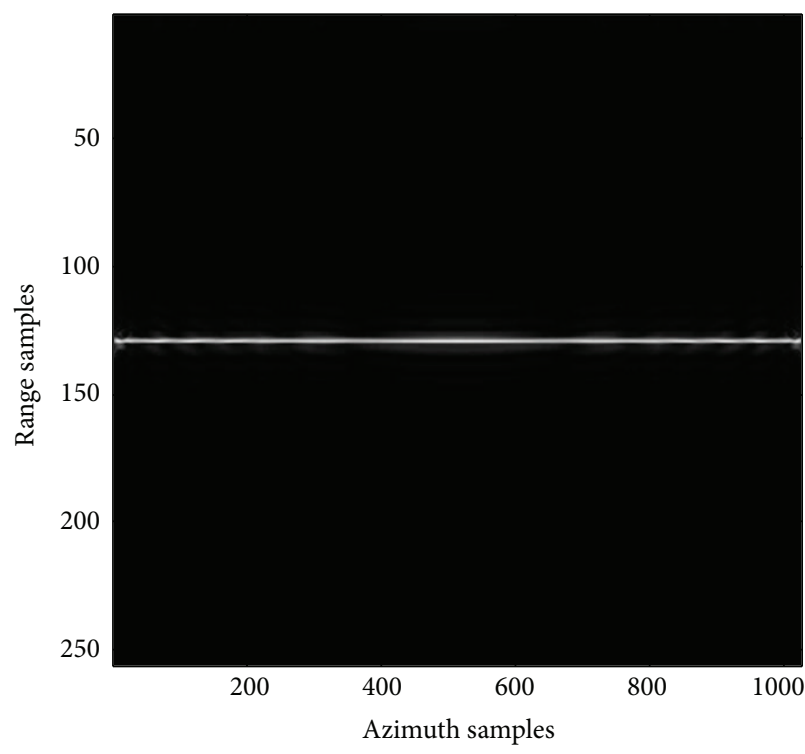

(c)

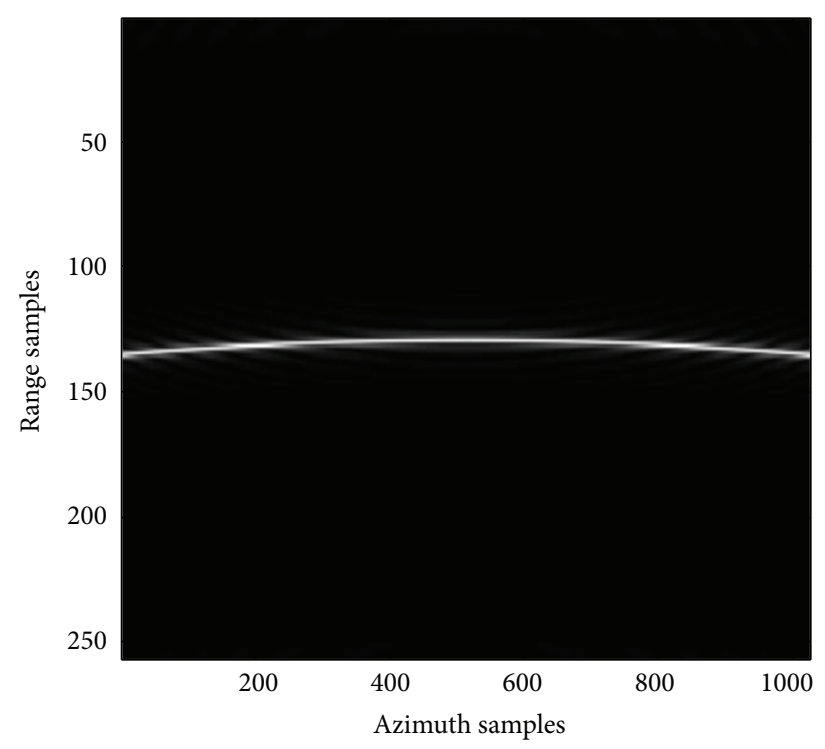

(b)

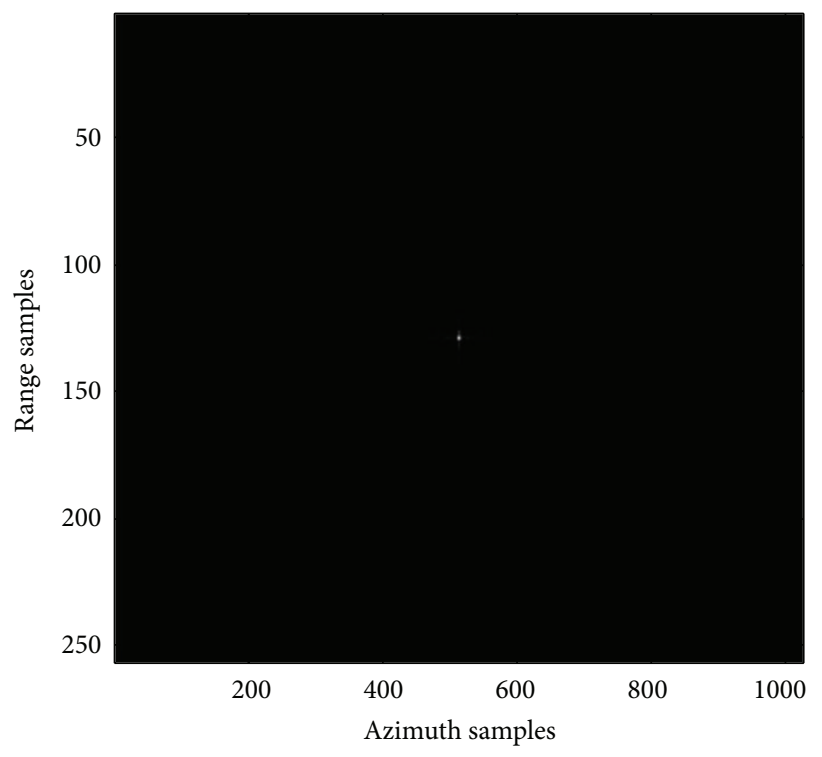

(d)

FIGURE 6: RDA validation for the point target. ((a) The real part of the raw signal; (b) the range compression results of echo signal; (c) the range-Doppler image; (d) the SAR image.)

2.5. Implementation of the Proposed Method. Based on the theoretical analysis in the previous chapter, the block scheme of the proposed echo generation and image simulation method for SAR is shown in Figure 7.
Consequently, the basic steps are outlined as follows:

(1) Set the SAR system parameters and the motion parameters, including bandwidth, sampling rate, and pulse repetition frequency. 


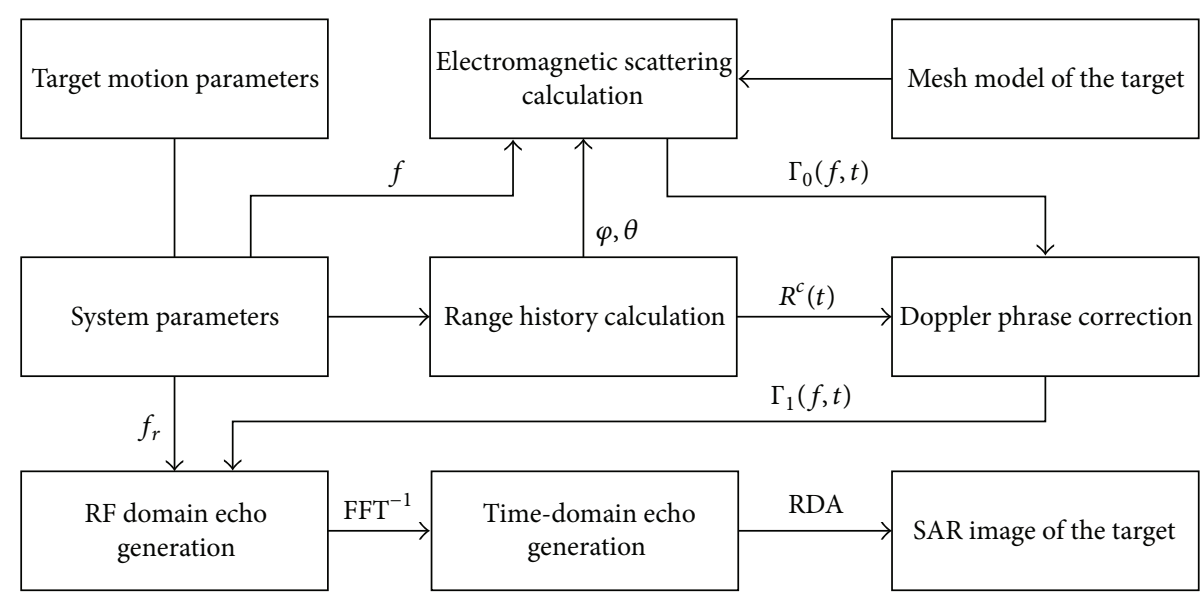

FIGURE 7: Block scheme of the simulation method.

TABLE 1: Simulation parameters.

\begin{tabular}{lccc}
\hline Initial distance & $10 \mathrm{~km}$ & Pitching angle & $90 \mathrm{deg}$ \\
Pulse duration & $1 \mathrm{us}$ & Squint angle & 0 deg \\
Carrier frequency & $1 \mathrm{GHz}$ & Pulse bandwidth & $100 \mathrm{MHz}$ \\
Number of the azimuth samples & 1024 & Number of the range samples & 256 \\
Pulse repetition frequency & $200 \mathrm{~Hz}$ & Fast time sampling rate & $120 \mathrm{MHz}$ \\
\hline
\end{tabular}

(2) Get the 3D model and the mesh model of the target. To ensure electrical connectivity, triangles must therefore share an edge. Similarly, segments must connect to other segments at nodes or to mesh triangles at vertices.

(3) For range history analysis according to the systems parameters and motion parameters, calculate the range history along with the azimuth and the pitching angle of the target relative to the SAR platform.

(4) Calculate the corresponding RCS matrix using the method described in the second part of the Section 2.2.

(5) Calculate the echo data with the scattering characteristic of the target in the frequency domain and then get the original time-domain echo data through inverse FFT.

(6) Finally, the output raw signal is subsequently processed by RD algorithm to obtain the SAR images, in order to prove the validity of the simulator.

\section{Simulations Results}

3.1. Simulations and Results. Raw signals simulation, relevant first to single scattering point and then to complex target, is now presented in this section to test the effectiveness of the simulator we proposed.

3.1.1. Point Target Verification. Firstly, in order to show the precision of our algorithm, we consider a fixed single point scatterer placed at the center of the irradiation belt. For such a scatterer, the SAR raw signal can be exactly computed directly in time domain. Accordingly, it is possible to compare the raw signal simulated via the approach we proposed to the exact one. The main parameters are listed in Table 1.

First of all, the simulated SAR raw data using TD method and our RFAT method are depicted in Figures 8(a) and 8(b). Two types of raw data look quite like each other, which confirm the effectiveness of our proposed algorithm.

To compare the raw data results clearly, the central signals of both the range direction and the azimuth direction are shown, respectively, in Figures $8(\mathrm{c})$ and $8(\mathrm{~d})$, which are extracted from Figures 8(a) and 8(b) signal and intuitively reflect the linear frequency modulation (LFM) characteristic. By contrast, we can observe that the two lines almost superposed each other, which proves the accuracy of the proposed method.

3.1.2. Complex Target Simulation. A plane model, with the size of $7.4 \mathrm{~m} \times 8.8 \mathrm{~m} \times 1 \mathrm{~m}$ shown in Figure 9, is utilized as a complex target to generate its raw data in this section. Besides, the monostatic case, high-speed case, and bistatic case are all implemented.

(a) Monostatic SAR Cases. First of all, in the case of rectilinear motion, consider the same typical X-band missile-borne SAR system of Figure 1, whose main parameters are listed in Table 2. It should be noted that, in this part of simulation, the $x$-axis is defined as the azimuth direction, the $y$-axis represents the range direction, and the $z$-axis is the vertical direction. 


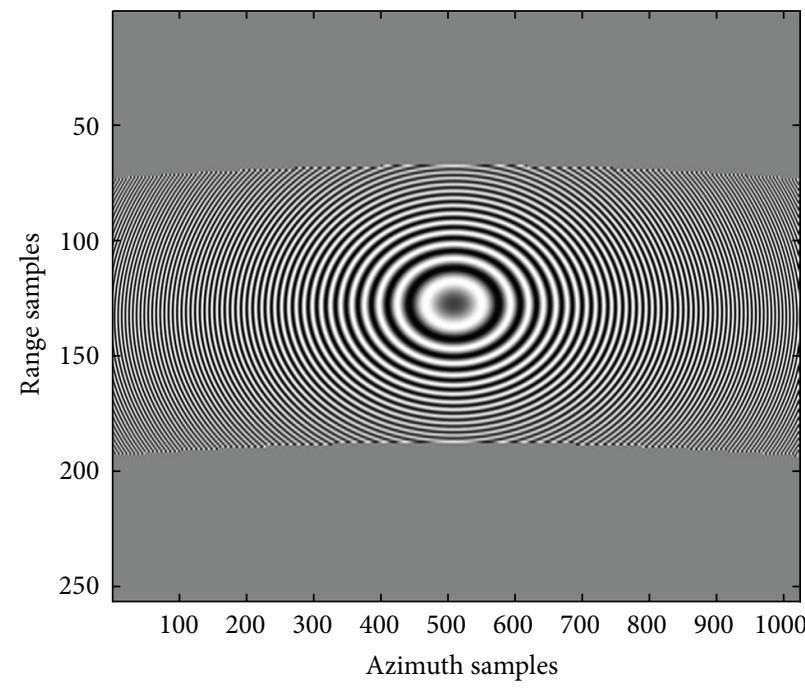

(a)

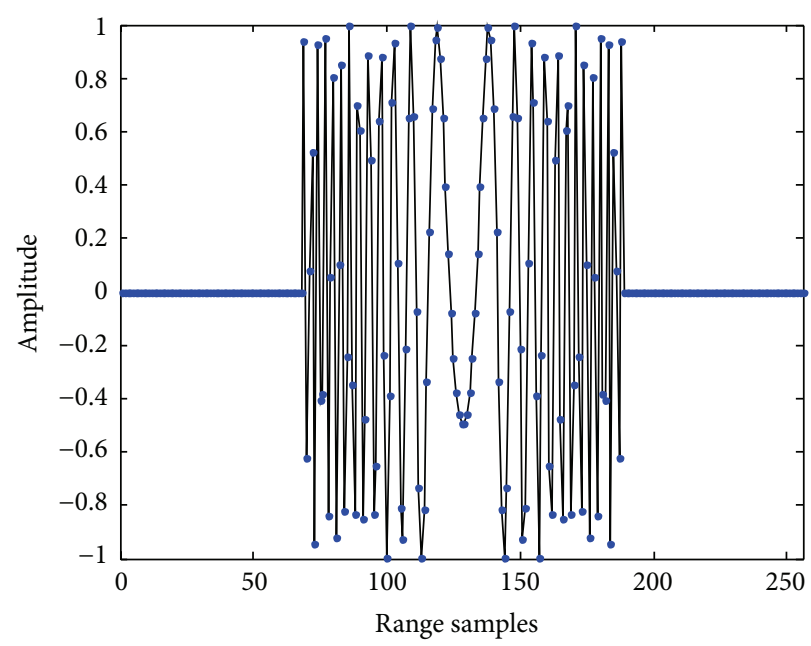

- TD method

- Our method

(c)

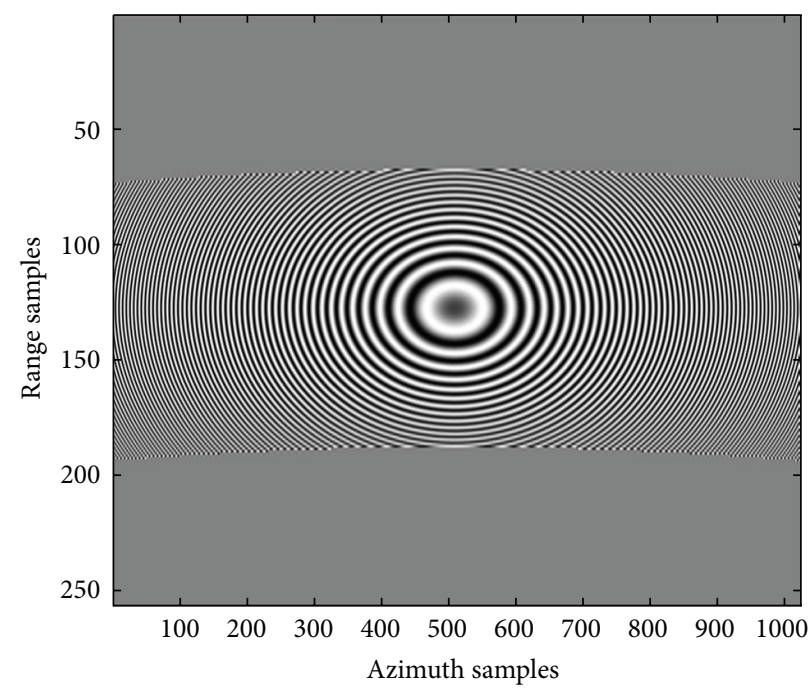

(b)

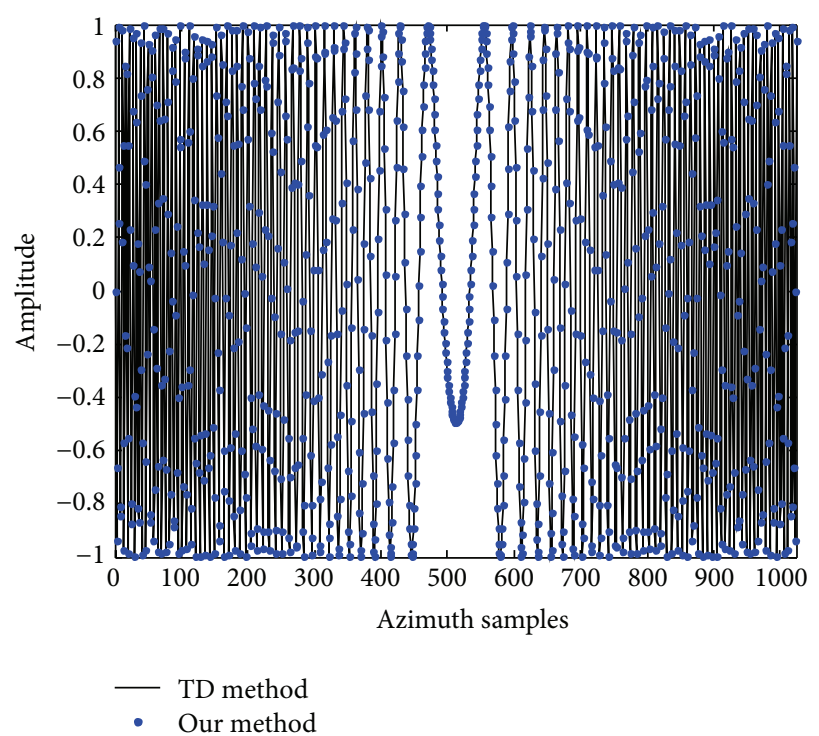

(d)

FIGURE 8: Simulated SAR raw data using different methods: (a) TD method; (b) our method; (c) range cut; (d) azimuth cut.

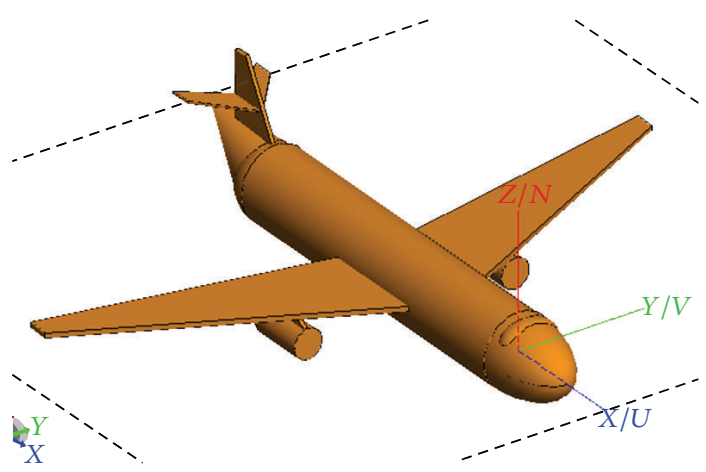

FIGURE 9: The 3D model of the plane. 
TABLE 2: Simulation parameters.

\begin{tabular}{lccc}
\hline Initial distance & $2.0 \mathrm{~km}$ & Pitching angle & $75 \mathrm{deg}$ \\
Pulse duration & $0.4 \mathrm{us}$ & Squint angle & $10 \mathrm{deg}$ \\
Carrier frequency & $10 \mathrm{GHz}$ & Pulse bandwidth & $600 \mathrm{MHz}$ \\
Synthetic aperture time & $0.3584 \mathrm{~s}$ & Antenna length & $0.98 \mathrm{~m}$ \\
Azimuth samples number & 163 & Range samples number & 252 \\
Relative velocity vector & $(150,0,0) \mathrm{m} / \mathrm{s}$ & Relative acceleration vector & $(0,0,0) \mathrm{m} / \mathrm{s}^{2}$ \\
\hline
\end{tabular}

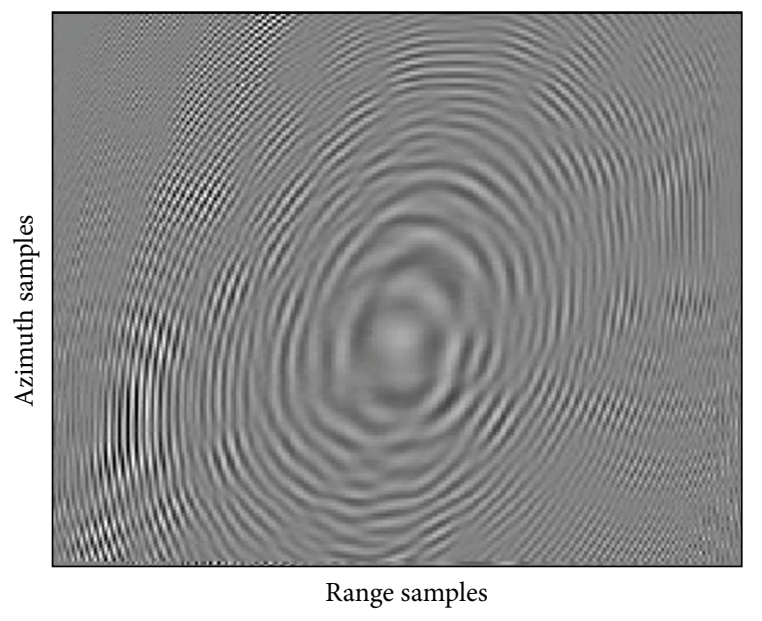

(a)

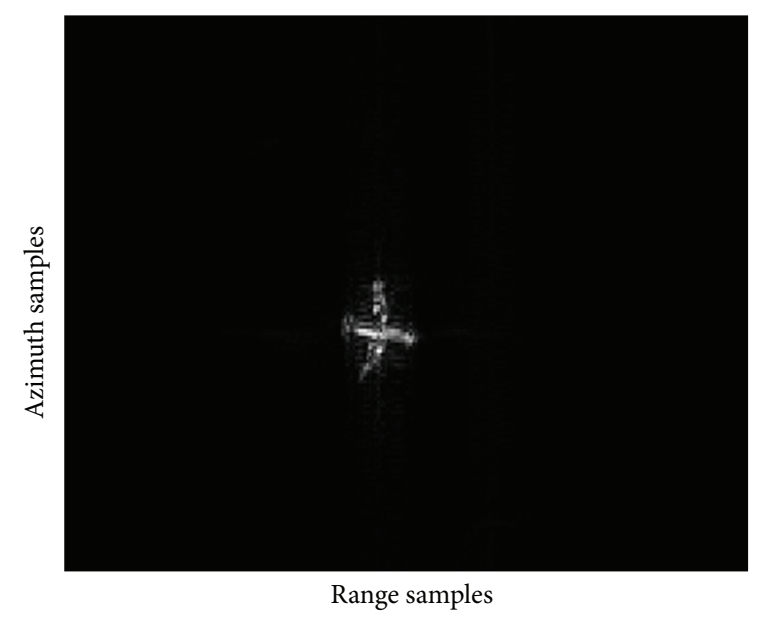

(b)

Figure 10: The simulated echo (a) and image (b) results of the plane model in the case of uniform linear motion.

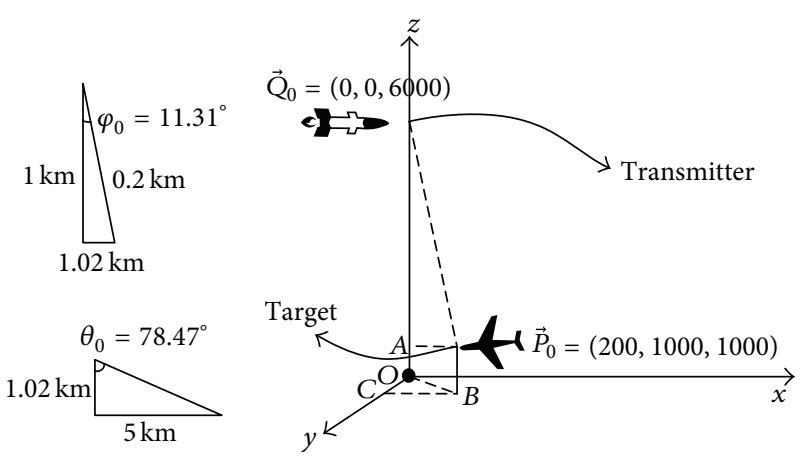

FIGURE 11: The trajectory diagram in the case of nonlinear motion.

According to the basic theory of SAR, the distance resolution and the azimuth resolution after pulse compression are, respectively, $0.25 \mathrm{~m}$ and $0.5 \mathrm{~m}$.

The simulation results are shown in Figure 10. Between them, (a) is the real part of the generated raw data and (b) is the obtained SAR image, which clearly reflects the structure information of the aircraft including the fuselage and wings, and the plane in the image tilts up to 10 degrees which matches well with the actual posture.

Then, in the case of curvilinear motion, the curve track is shown in Figure 11.

It can be seen from Figure 11 that the missile platform locates at $(0,0,6000)$ initially, and the plane target is at $(200,1000,1000)$. By solving the triangle $A P_{0} Q_{0}$ and $O C B$, we obtain the initial azimuth angle and pitching angle which, respectively, are $\varphi_{0}=11.31^{\circ}$ and $\theta_{0}=78.47^{\circ}$. Besides, the relative velocity is set as $\vec{v}_{p q}=(100,20,10) \mathrm{m} / \mathrm{s}$, and the relative acceleration is set as $\vec{a}_{p q}=(10,10,10) \mathrm{m}^{2} / \mathrm{s}$.

The results of echo signal and SAR image are, respectively, shown in Figure 12. It is easy to distinguish a plane shape in Figure 12(b). By comparing Figures 10(b) and 12(b), we can see that, in the case of curve track, the simulated SAR image is fuzzier; this is due to the nonuniform sampling caused by the nonideal movement.

(b) High-Speed Cases. We also performed a series of highspeed missile-borne SAR simulation experiments. The system also works in $\mathrm{X}$ band. The bandwidth of the transmitted chirp signal is $1 \mathrm{GHz}$; PRF is $2000 \mathrm{~Hz}$. In the vertical direction, the relative height is initially $6000 \mathrm{~m}$ and the relative velocity is $0 \mathrm{~m} / \mathrm{s}$, while the acceleration is controlled to be $20 \mathrm{~m} / \mathrm{s}^{2}$. In the azimuth direction, the relative velocity is set to be $1000 \mathrm{~m} / \mathrm{s}$, and both the initial distance and the acceleration are zero. Finally, in the range direction, the relative velocity and acceleration are also set to be zero. At the imaging moment, the pitching angle and the azimuth angle are about $50^{\circ}$ and $30^{\circ}$, respectively.

Figure 13 shows the simulated SAR echo result and image result. Between them, (a) is the real part of the raw data and (b) is the SAR image obtained by processing the raw data with the $\mathrm{RD}$ algorithm. It can be seen from Figure 15(b) that a plane was flying in the direction of the 30 degrees. 


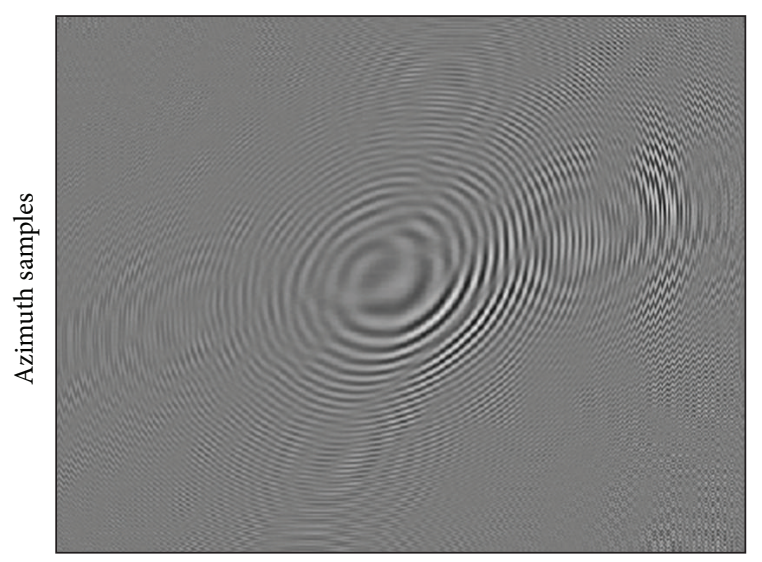

Range samples

(a)

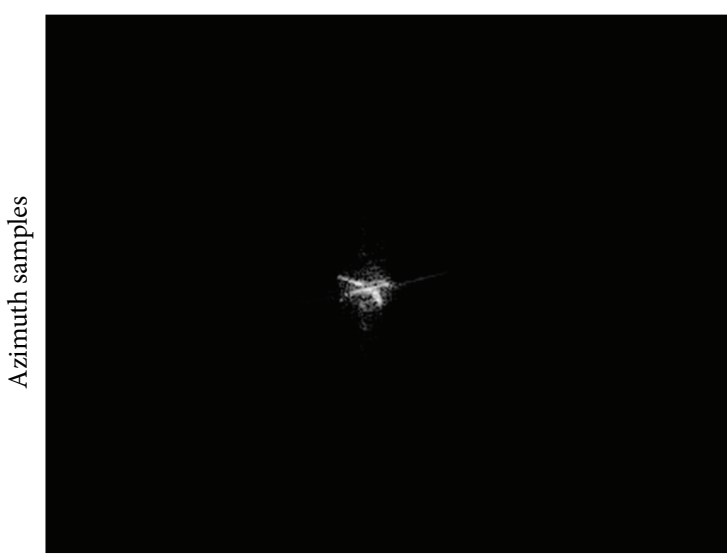

Range samples

(b)

FIGURE 12: The simulated echo (a) and image (b) results of the plane model in the case of nonlinear motion.

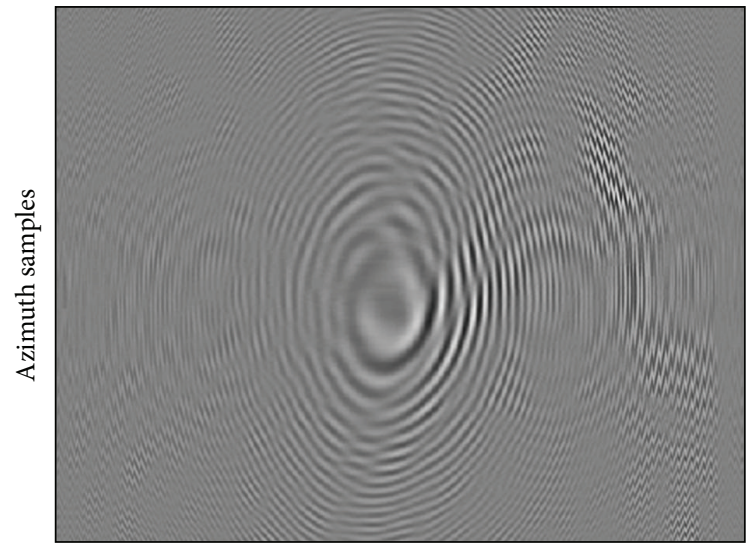

Range samples

(a)

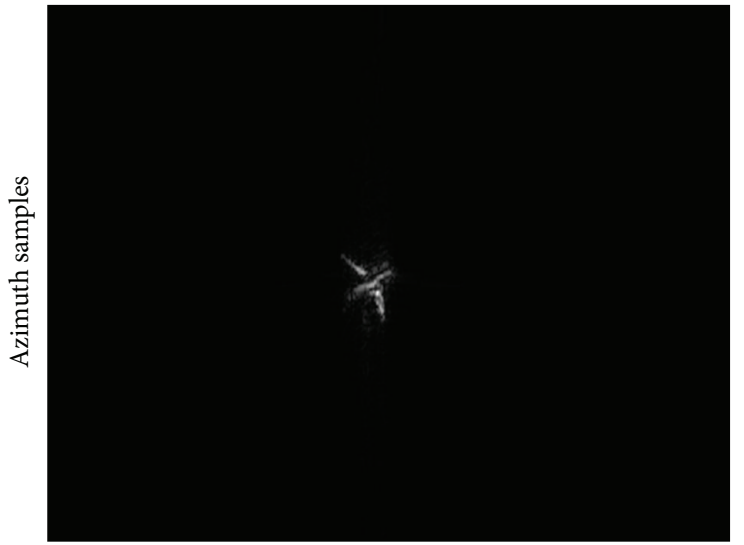

Range samples

(b)

Figure 13: The simulated echo (a) and image (b) results of the plane model in the case of high- speed.

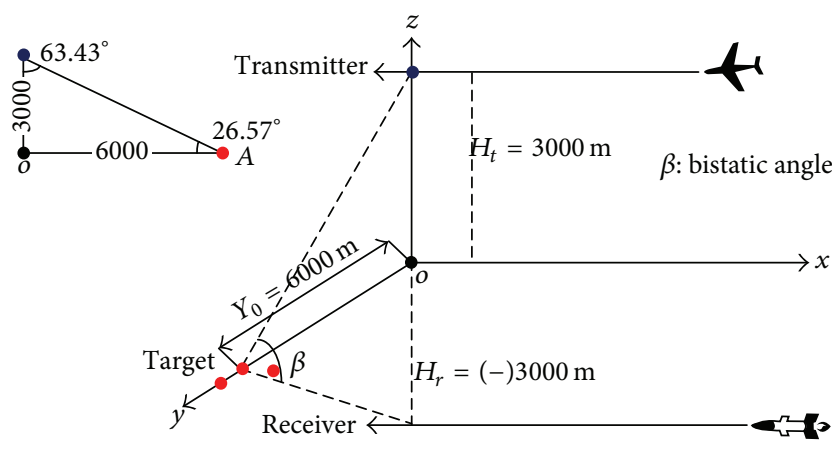

FIGURE 14: The geometry diagram in the simulation of bistatic case.

(c) Bistatic SAR Cases. Finally, we implemented a missileborne bistatic SAR simulation. In this simulation, the receiver and transmitter travel along different but parallel tracks with constant and equal velocities which are controlled to be
TABLE 3: The velocity and location of the three point scatterers.

\begin{tabular}{lcc}
\hline Point scatterer & Location & $\begin{array}{c}\text { Velocity along the } \\
x \text {-axis direction }\end{array}$ \\
\hline A & $(0,6000,0)$ & $50 \mathrm{~m} / \mathrm{s}$ \\
B & $(0,6010,0)$ & $50 \mathrm{~m} / \mathrm{s}$ \\
C & $(10,6000,0)$ & $50 \mathrm{~m} / \mathrm{s}$ \\
\hline
\end{tabular}

$150 \mathrm{~m} / \mathrm{s}$, and they share the same squint angle of $0^{\circ}$. The bistatic angle achieves $53.14^{\circ}$, which means that the system has quite a high bistatic degree as well. The heights of the transmitter and receiver both are $3000 \mathrm{~m}$, respectively. The geometry configuration is shown in Figure 14.

The simulated scene consists of three point scatterers, whose locations and velocity are shown in Table 3. Scatterer A locates in the center of the scene; A and B locate in the same instantaneous Doppler frequency but different range 


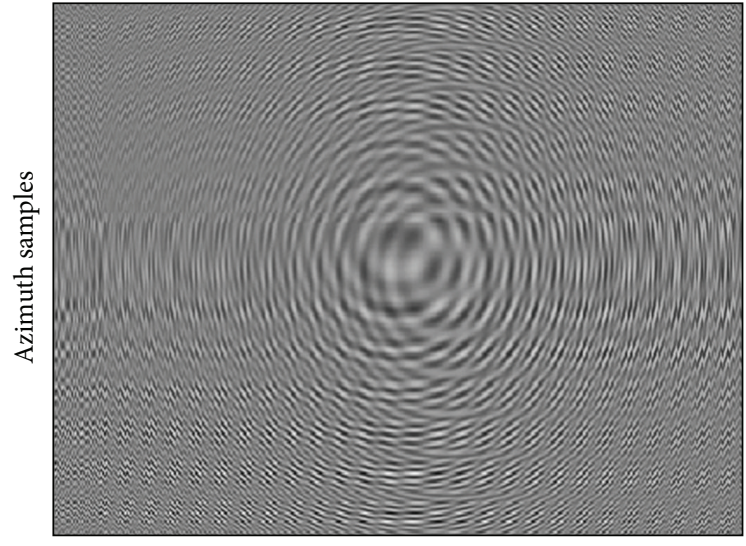

Range samples

(a)

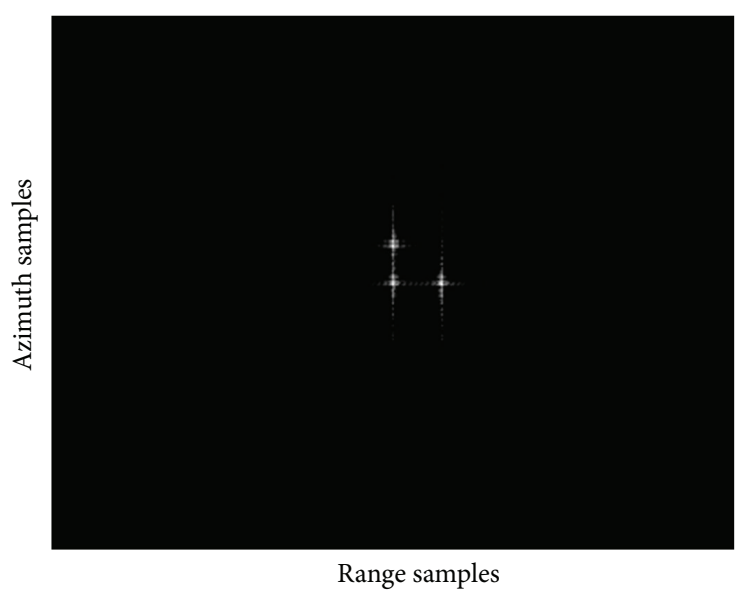

(b)

FIGURE 15: The simulated echo (a) and image (b) results of the plane model in the case of bistatic SAR.

cells, while $\mathrm{A}$ and $\mathrm{C}$ have the same range cell but different instantaneous Doppler frequency.

Figure 15(a) shows the real part of the simulated raw data matrix; Figure 15(b) shows the imaging result where we can see that all the point scatterers are well focused at the expected position.

3.2. Computational Efficiency Analysis. Compared with the TD method, the proposed simulator has the advantage of computational efficiency which can be expressed as

$$
C_{\mathrm{Tol}}=N_{r} N_{a} C_{R}+\frac{1}{2 N_{r} N_{a} \log _{2}\left(N_{r}\right)}+N_{r} N_{a}
$$

where $1 / 2 N_{r} N_{a} \log _{2}\left(N_{r}\right)$ originates the IFFT operation, $N_{r} N_{a}$ originates the dot product of the matrix, and $C_{R}$ represents the complexity for computing single frequency RCS of the whole target which determines the whole processing time to a large extent. For the above-mentioned plane model in Figure 9 (with the size of $7.4 \mathrm{~m} \times 8.8 \mathrm{~m} \times 1 \mathrm{~m}$ ), the single frequency RCS calculating time reaches to $2.54 \mathrm{~s}$ in the case of a computer with 8 cores. In fact, we conduct our simulation along with 10 programs in a computer with 16 cores; in this case, to obtain the simulation results of Figure 10, the total processing time is about

$$
2.54 \times 252 \times 163 / 10 / 2 / 3600 \approx 1.45 \mathrm{~h} .
$$

It is clear that the proposed method is much more computationally efficient than the TD method; however, as a tradeoff, it is more time consuming than the $2 \mathrm{D}$ method. In particular, the improving calculation condition like the cluster technology could make the simulation of larger targets possible in a more reasonable time.

\section{Conclusion}

To support SAR system design for target recognition, a novel RFAT SAR simulation method which realized the transformation from geometric model to SAR image has been presented. The method not only is faster compared with the TD simulator but also can be useful in applications where a 2D simulator cannot be used. The simulation results prove that the wide radar scattering characteristic of target has a great impact on the SAR imaging. Different target postures and different SAR system can cause a huge difference in the SAR image, which further demonstrates the difficulty of high-resolution SAR image interpretation. Thus, a lot more research is needed in the SAR imaging mechanism.

\section{Competing Interests}

The authors declare that there are no competing interests regarding the publication of this paper.

\section{Acknowledgments}

The work was supported by "The Fundamental Research Funds for the Central Universities" (no. NS2016040).

\section{References}

[1] I. G. Cumming and F. H. Wong, Digital Processing of Synthetic Aperture Radar Data: Algorithms and Implementation, Artech House, Norwood, Mass, USA, 2005.

[2] G.-O. Glentis, K. Zhao, A. Jakobsson, H. Abeida, and J. Li, "SAR imaging via efficient implementations of sparse ML approaches," Signal Processing, vol. 95, no. 2, pp. 15-26, 2014.

[3] B. Deng, X. Li, H. Wang, Y. Qin, and J. Wang, "Fast raw-signal simulation of extended scenes for missile-borne SAR with constant acceleration," IEEE Geoscience and Remote Sensing Letters, vol. 8, no. 1, pp. 44-48, 2011.

[4] A. Mori and F. De Vita, "A time-domain raw signal simulator for interferometric SAR," IEEE Transactions on Geoscience and Remote Sensing, vol. 42, no. 9, pp. 1811-1817, 2004.

[5] S. Cimmino, G. Franceschetti, A. Iodice, D. Riccio, and G. Ruello, "Efficient spotlight SAR raw signal simulation of 
extended scenes," IEEE Transactions on Geoscience and Remote Sensing, vol. 41, no. 10, pp. 2329-2337, 2003.

[6] L. Yang, W. Yu, S. Zheng, and L. Zhang, "Efficient bistatic SAR raw signal simulator of extended scenes," International Journal of Antennas and Propagation, vol. 2014, Article ID 130784, 9 pages, 2014.

[7] Y. Wang, Z. Zhang, and Y. Deng, "Squint spotlight SAR raw signal simulation in the frequency domain using optical principles," IEEE Transactions on Geoscience and Remote Sensing, vol. 46, no. 8, pp. 2208-2215, 2008.

[8] Y. Tian, S. Guo, Y. Wang et al., "A novel geo-SAR echo simulation method," in Proceedings of the 10th European Conference on Synthetic Aperture Radar (EUSAR '14), pp. 1-4, VDE, Berlin, Germany, June 2014.

[9] B.-N. Wang, F. Zhang, and M.-S. Xiang, "SAR raw signal fast algorithm in mixed domain," Journal of Electronics and Information Technology, vol. 33, no. 3, pp. 690-695, 2011.

[10] G. Franceschetti, R. Guida, A. Iodice, D. Riccio, and G. Ruello, "Efficient simulation of hybrid stripmap/spotlight SAR raw signals from extended scenes," IEEE Transactions on Geoscience and Remote Sensing, vol. 42, no. 11, pp. 2385-2396, 2004.

[11] G. Franceschetti, A. Iodice, S. Perna, and D. Riccio, "SAR sensor trajectory deviations: fourier domain formulation and extended scene simulation of raw signal," IEEE Transactions on Geoscience and Remote Sensing, vol. 44, no. 9, pp. 2323-2333, 2006.

[12] X. Liu, L.-R. Zhang, and Y. Zhou, "2-D Fourier domain fast algorithm for UWB ground penetrating SAR raw signal simulation," Journal of Chang'an University (Natural Science Edition), vol. 34, no. 4, pp. 109-114, 2014.

[13] X. Liu, C. Zeng, J. Wang, and W. Zhao, "Improved method for SAR echo signal simulation using two-dimensional frequency domain transformation," Journal of Xidian University, vol. 40, no. 3, pp. 42-49, 2013.

[14] R.-J. Li, K.-F. Ji, H.-X. Zou et al., "Simulation of SAR imagery of target based on electromagnetic scattering characteristic computation," Radar Science and Technology, no. 5, pp. 395-405, 2010.

[15] J. Qin and Z. M. Zhang, "Implementation and optimization of SAR echo simulation based on GPU," Science Technology and Engineering, vol. 14, no. 3, pp. 85-89, 2014.

[16] G. Franceschetti, A. Iodice, D. Riccio, and S. Perna, "Efficient simulation of airborne SAR raw data of extended scenes," IEEE Transactions on Geoscience and Remote Sensing, vol. 44, no. 10, pp. 2851-2860, 2006.

[17] Z. Meng, Y. Li, C. Li, M. Xing, and Z. Bao, "A raw data simulator for Bistatic Forward-looking High-speed Maneuveringplatform SAR," Signal Processing, vol. 117, pp. 151-164, 2015.

[18] R. G. Kouyoumjian, L. Peters, and D. T. Thomas, "A modified geometrical optics method for scattering by dielectric bodies," IEEE Transactions on Antennas and Propagation, vol. 11, no. 6, pp. 690-703, 1963.

[19] X. Weijie and W. Xiaojie, "SAR image simulation and verification for urban structures," International Journal of Electronics, vol. 103, no. 2, pp. 247-260, 2016.

[20] K. M. Mitzner, "Incremental length diffraction coefficients," Tech. Rep. AFAL-TR-73-296, Aircraft Division, Northrop Corporation, 1974.

[21] C. Ning, C.-Z. Dong, J. Huang, and X.-Y. Zhang, "Simulation of dynamic RCS data on space flight target," Computer Engineering and Design, vol. 35, no. 4, pp. 1367-1371, 2014. 


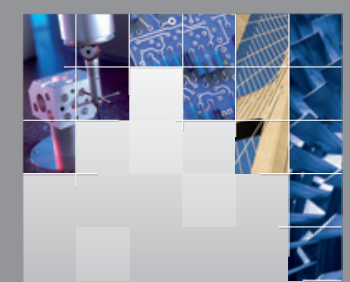

\section{Enfincering}
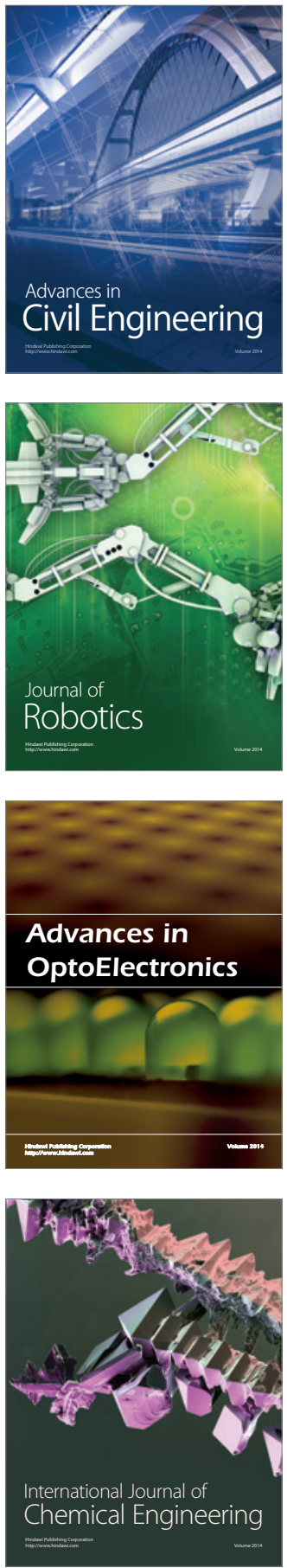

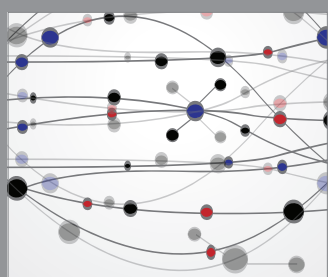

The Scientific World Journal

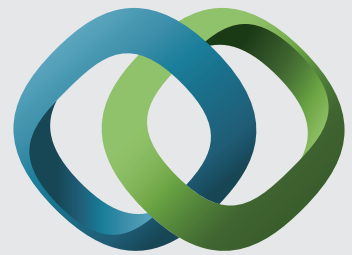

\section{Hindawi}

Submit your manuscripts at

http://www.hindawi.com
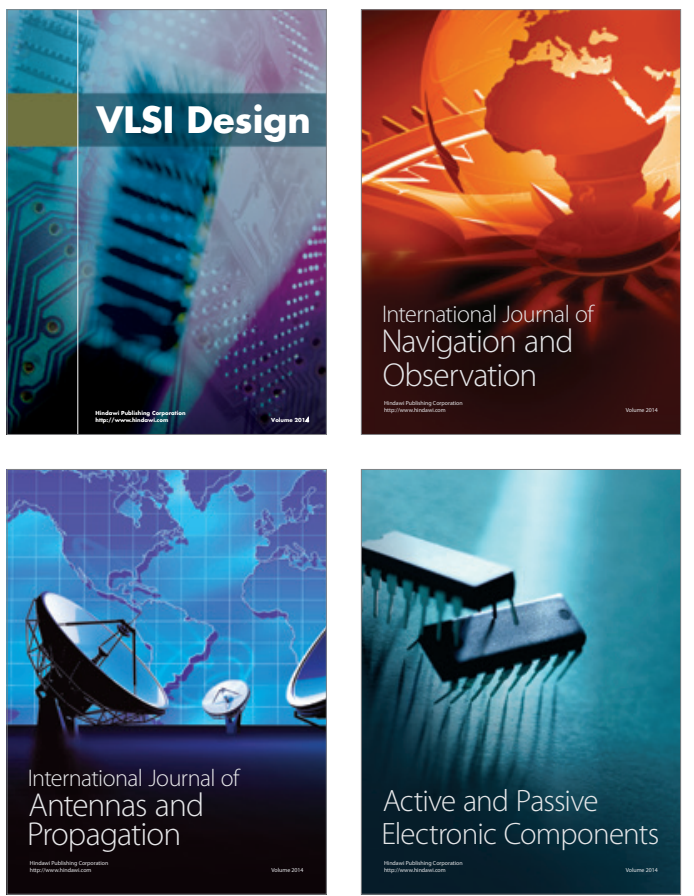
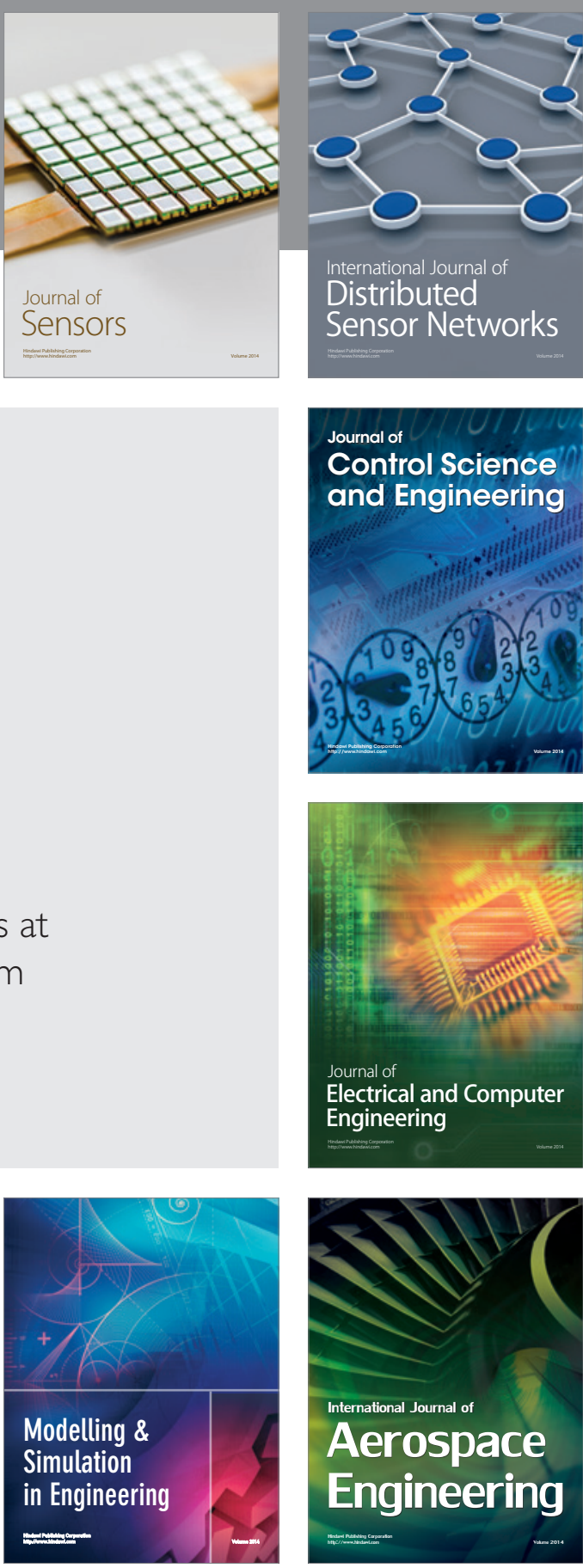

International Journal of

Distributed

Sensor Networks

Journal of

Control Science

and Engineering
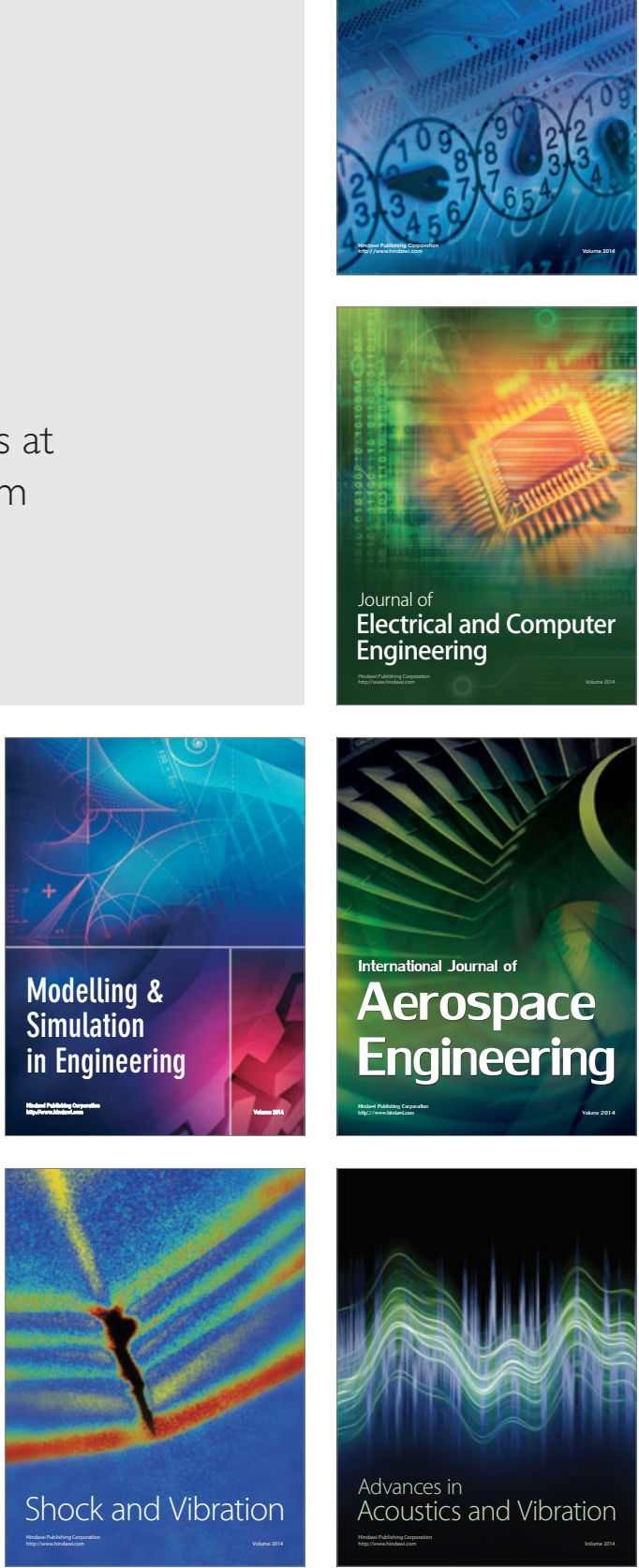